Open Access

\title{
The evolvement of family intergenerational relationship in transition: mechanism, logic, and tension
}

\author{
Jinqun Shi
}

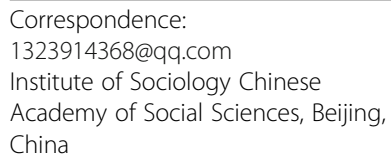

\begin{abstract}
Based on diversified intergenerational living arrangements in urban China, this paper studies the characterization and formation mechanism of intergenerational family relationships in the Chinese society. The results show that the subject of an intergenerational relationship seeks individual freedom, while simultaneously being subjected to the everyday life logic of family responsibilities, including filial piety, and the limitation of social support with regard to elderly care and child rearing. The intergenerational family relationship thus becomes the last bastion of the individual to resist risks. Most people involved in intergenerational relationships try to balance the tensions between social structure and the individual by selecting different forms of intergenerational living arrangements, which form unique evolving family intergenerational relations.
\end{abstract}

Keywords: Intergenerational family relations, Structure, Individual, Evolvement

The stem family and the nuclear family have always been, respectively, the primary and traditional forms of Chinese intergenerational relationship-after the children are grown, most children form their own core family and leave one child living with parents to form a stem family. These intergenerational forms are relatively stable. In these stable intergenerational forms, adult children and their parents maintain a strong and stable connection, forming an intergenerational family relationship with Chinese characteristics. However, over the past few decades, the macro environment of the family has changed as a result of China's enormous political, economic, cultural, social, and demographic changes; the size of the family has shrunk; and the family structure has been reconstructed. This is bound to bring new challenges to traditional family intergenerational relations (Ma Chunhua et al. 2013).

The form of intergenerational relationship is one of the most direct and objective dimensions of observing intergenerational family relationships (Bengtson and Roberts 1991). However, in the study of intergenerational relations, classical family modernization theory has had an enduring impact, and the intergenerational relationship form in China often categorized according to the classic division of "extended-stem-unclear" as a basic reference. Scholars have analyzed changes in the proportion of stem family and nuclear family based on sample surveys of typical areas or large population census data, and have taken the changes as a basis for studying the classical family modernization theory.

(c) The Author(s). 2017 Open Access This article is distributed under the terms of the Creative Commons Attribution 4.0 International License (http://creativecommons.org/licenses/by/4.0/), which permits unrestricted use, distribution, and reproduction in any medium provided you give appropriate credit to the original author(s) and the source, provide a link to the Creative Commons license, and indicate if changes were made. 
However, the author finds in a field survey that there are actually a variety of intermediate states of intergenerational relationship forms that fall between nuclear families and stem families. The intergenerational forms of these intermediate states have often been overlooked in previous studies of family structure. For example, with regard to intergenerational living arrangements, a phenomenon of great concern in recent years has been elderly parents living with their adult children, showing that the traditional form of stem family is in decline. But the truth may be that the decline in the proportion of parents and adult children living together only reflects one aspect of the phenomenon, and what is behind the decline in this proportion is diversification of intergenerational living arrangements.

For example, when elderly parents need to be taken care of because of illness or for other reasons, or adult children need assistance in life due to encountering difficulties, adult children and parents may re-compose to form a temporary stem family for mutual benefit. As economic conditions have improved and individualistic values are strengthened, the proportion of elderly parents living close to their adult children is also rising. Traditionally, in principle elderly parents live together with a married son. Nowadays, elderly parents may live together with either adult daughters or adult sons, showing a diversity of living forms. In addition, if elderly parents have many children, they may also periodically live with different children in turn or live with different children, respectively. These phenomena indicate diversity and mobility in the living arrangements of elderly parents and adult children. Especially during China's transitional period, structural and individual factors affect and constrain each other, making the forms of intergenerational relationship more diverse and complex.

Foreign scholars have noted that there are different forms of intergenerational relations that exist in modern society. For example, in studies of Japanese intergenerational relations, there have been theoretical debates on family separation, family separation existence, and amendatory lineal family. (Song 2001). In China's transition period, the study of intergenerational relations should go beyond the classical classification framework and shift from the traditional quantitative typological analysis of family structure to the practical analysis of family life (Yao 2012). Based on this idea, this paper attempts to examine the characteristics and the formation mechanism of intergenerational relations in China's transitional period through the case study of diversified intergenerational living arrangements in modern cities.

\section{Literature review and research methods}

The bridge between structure and individual in intergenerational relationship studies Structuralism in the early study of intergenerational relations

In the development of social history, although the intergenerational family relationship always has had an important influence on the individual and society, scholarly attention and scientific research on this topic only began following the restructuring of social structure (including family structure) brought about by industrialization and urbanization. Under the influence of modernization theory and structural functionalism, the classical modernization theory on family represented by W. J. Goode and Burgess has had far-reaching influence on research in various fields of family, and the family intergenerational relationship was no exception. One of the key points of the classical family modernization theory of family 
intergenerational relations is that, with the further advance of industrialization and modernization, the extended family or joint family, which is closely related to kinship group, will gradually be replaced by a relatively small family grouping (nuclear family or couple family). This change follows a linear sequence and a linear development model. The driving force of this change to family structure is mainly the industrialization. Industrialization has led to the disintegration of the extended family, while the nuclear family and couple family are most suitable for the needs of industrialization. The isolating effect of the nuclear family form is the inevitable outcome of highly economically developed societies. Job opportunities in various regions of a city create the possibility of occupational and geographical mobility. In order to better adapt to the modern economy's demand for labor mobility and labor skills, adult children have to widen their geographic and social distance from their parents, or economic and social development will stagnate. The development of education, health care, and mutual aid organizations have further reduced the dependency of people on kinship networks (Parsons 1949; Goode 1982).

Obviously, in classical modernization theory on family, the level of economic development is a decisive factor in the development of patterns of intergenerational relations. This theoretical interpretation of structural functionalism, however, fails to explain the diversified changes in family patterns in developing countries since the 1960s.

Since the 1970s, scholars have questioned the core arguments of classical modernization theory on family from both historical and empirical perspectives (Laslett 1972; Shorter 1977). Through a number of comparative studies, scholars have found that differences in patterns of intergenerational relations between different societies are not determined by the actual levels of economic development of these societies. From a vertical perspective, the European family had the so-called modern family features before industrialization and urbanization, that is, the structure of the nuclear family predates industrialization and urbanization; from a horizontal perspective, the family pattern for different countries with different economic levels do not occur in a linear sequence, but the intergenerational relationship pattern presents diverse and complex features. In this context, cultural difference has been introduced as another important explanatory variable in family studies, forming another theoretical-explanatory dimension of the evolution of intergenerational relations patterns, namely, the theory of cultural influence (Tang 2010).

\section{The integration of the individual's perspective in the study of intergenerational relations}

The theory of cultural influence enhanced the theoretical explanatory power of the diversity of intergenerational relations. But as with classical modernization theory and its emphasis on economic development, both theories are interpretations based on macrolevel factors that analyze and explain changes in intergenerational relations from the perspective of macro-structures. The important mediating force in intergenerational relations, i.e., the individual's own initiative in choosing or shaping the intergenerational relations is neglected, leaving unexplained many micro-level phenomena. For example, macro-social structure and local cultural background are primary contributors to the social environment all the families face, but existing different patterns of intergenerational relations persist. One important reason is that current theories ignore the intergenerational interaction process at the microscopic level and the individual's own initiative in choosing or shaping the intergenerational relationship. With the theoretical reflection on the grand narrative of industrialization and modernization, family theory of the late twentieth century increasingly emphasized the effect of family on the 
individual, rather than the significance of family to society. Today study on intergenerational relationships has changed from the static and overall analysis to increased focus on more dynamic and individualized cases (Tang 2010).

Especially under the influence of the second modernity, family life has become a cause of self-identity and self-creation. Individuals are more concerned about the realization of their expressive needs, and norms no longer take precedent over individual behavior, but instead are constantly being defined and changed by individuals (Cherlin 2008). The second modernity has disembedded individuals from the traditional family system and kinship relationships, eliminating the linear and standardized life path (Beck and Beck-Gernsheim 2011).

Under the influence of individualization theory, researchers represented by the German scholar Lüscher introduced the individual perspective into the study of intergenerational relations. She argues that when people seek the significance of personal and social relationships, they swing between the individual level of feelings, thoughts, wishes, and structural factors. Therefore, she advocates focusing on the individual dimensions of intergenerational relations. But influenced by post modernism and feminist theory, this theoretical school emphasized the contradictions in intergenerational relations, not limited to the individual and the conflicting side, which has escaped the trap of over-emphasizing self-reflexivity, deinstitutionalization and detraditionalization, and thus, the structure and the individual are unified.

One of the core arguments of this theoretical school is that intergenerational relations are the result of continuous game and consultation between structure and self-subjectivity; as a result. Neither the initiative of the subject of the intergenerational relationship should not be ignored nor the restriction effect from the role and norms at the macro-level on the intergenerational relationship should be ignored. This theory pays attention to the game between the subject and the structure of the intergenerational action, the dynamic mechanism of the conversion of unity and conflict, and the process and strategy of family members in resolving contradictions. Furthermore, the theory focuses on the process and the contextual factors of actual consultation and interaction between intergenerational relationships, remedying the micro-defects of classical modernization theory on family and the theory of cultural influence; meanwhile, it has expanded the sociological perspective in studying intergenerational relationships; and by combining macro-research and micro-research on intergenerational relationships, it has improved the theoretical explanatory power of current complex phenomenon of intergenerational relationships, and thus, it has become an important strategy for the study of intergenerational relations since the 1990s (Connidis and McMullin 2002; Bengtson et al. 2002; Lüscher 2002; Lüscher and Pillemer 1998).

\section{Relevant research on intergenerational family relationship in China}

Since the 1980s and 1990s, China's economic and social transformation has brought great changes to its society. Scholars of Chinese studies began to pay attention to the influence caused by this social transformation on intergenerational family relationships. However, since the concept and theory of modernization have long dominated the analysis of social changes in China, scholars at home and abroad more often use the analytical path of classic modernization theory to analyze changes to the intergenerational relations of Chinese families. Assuming there is a contradiction between tradition and modernity, then the changes to the intergenerational relations of Chinese families is following or will follow the direction described in classic modernization theory on 
family. That is, family size is shrinking and family structure is centralizing, and the horizontal marital axis is replacing the horizontal parental axis as the main one in the family relationhip. (Luo 2004; Liu 2000).

However, with further in-depth changes, facing the powerlessness of classic modernization theory on family to explain diverse family structures and complex family relations in modern Chinese society, the researchers began a profound reconstruction of this theory: does the isolated nuclear family and increasingly alienated adult child and his/her parents offer a true picture of the changes in the intergenerational relationship of Chinese urban families? In this process of reflection, some excellent research emerged, and this research has been helpful for help us to gain a better understanding of the intergenerational relations of Chinese families. For example, researchers have found that the nuclear family in China is not isolated; the relationships between adult children and their parents are even closer than before; and there have been "live off one's parents" (boomerang child/kid), "close intergeneration family relations," as well as "return to the stem family" relationships (Kang 2009; Shen 2013; Wang 2011; Ma Chunhua et al. 2013; Tang and Chen 2012; Xiao 2014). Some scholars have conducted preliminary analyses of these formal "regression to tradition". For instance, Kang Lan believes that China's traditional values and lived experiences made the intergenerational relationships in Chinese urban families a unique pattern that differs from the feedback model or relay model (Kang 2009); from the individual perspective, Shen Yifei and Yao Jun instead emphasize the reasons leading to this phenomenon and the importance of individuals to family structure and intergenerational relationships. They suggest that this form of "regression to tradition" is actually more of an embodiment of individualization in modern intergenerational relations (Shen 2013; Yao 2012).

Thus, it can be seen that scholars had noticed the impact of the transition period on traditional intergenerational family relations, and also tried to analyze the reasons for changes happened to intergenerational family relations from structural and individual aspects. But combined with the existing research and the author's survey, the author believes two aspects require further study: first, thoroughly grasping the complex phenomena of intergenerational relations. Although scholars have seen the complexity and diversity of intergenerational relations, currently the study of intergenerational relations is still limited to the classic classification of "living together" and "not living together," "stem," and "nuclear." For example, Chen Jieming has studied influential factors, determining that the better the economic conditions of parents and offspring, the lower the possibility of living together (Chen and Chen 2016). However, the author found during fieldwork that living together can be divided into a variety of situations; some are living together temporarily, some are living together long-term, some are living with the husband's parents, and some are living with the wife's parents. "Not living together" is also similar to the above situation. These different intergenerational living arrangements reflect different types of intergenerational relations. Modern intergenerational interaction is a dynamic and flexible process that requires us to conduct more in-depth analysis. Second, more analysis is needed of the formation mechanism of complex intergenerational relations in the transition period. Some scholars have noticed the particularity and diversity of Chinese intergenerational relations in the transition period and have attempted to explain the reasons from the perspective of individualization (Shen 2013; Yao 2012). However, the author also found during fieldwork that family 
intergenerational relationships in China's transition period is a process of unity and conflict. The explanation from the individual level is not enough, and the structural factors cannot be ignored either. Tang Can et al. pointed out that the intergenerational family relationship in the transition period is presenting another form, that is, mobile and temporary three-generation families are quite common in the life experience of Chinese families. It is a mutual relationship, and even if utilitarian factors exist, they typically derive from a set of life logics present in the Chinese culture of family responsibility, family obligation, and family love (Tang and Chen 2012).

To understand and analyze the intergenerational family relations during the transition period, we need to place it in the overall framework of the structure and the individual.

In this respect, the intergenerational theoretical school represented by Lüscher can provide some useful references. But this theoretical school only provides a broad framework and path of analysis. When placed in China's specific scenarios, especially within China's transition period and its special history and culture, what characteristics of intergenerational family relationships will appear? What is the decisive force that restricts the change of modern Chinese intergenerational family relations, and what is its inner logic? These need to be combined with China's specific situation for a more in-depth analysis (Fig. 1).

In view of this, and based on the diversified intergenerational living arrangements in Chinese cities at the present stage, this paper tries to explore the main characteristics of intergenerational family relations in China's transition period and analyzes the construction logic behind the individual and the social structure. Discussing the characteristics and the restraining mechanism can not only enrich the relevant theory of intergenerational relations, but also help to grasp the characteristics of the changes of intergenerational family relations in today's China. Further, this research may be helpful in the formulation of relevant family policies to actively respond to China's growing population aging problem and other related social problems.

\section{Methodology}

The materials used in this paper came from multiple sources: first, qualitative survey was conducted by the author in Guangzhou in 2008 over a period of 1 month. Guangzhou is a city with high degree of modernization, more developed market economy, and a higher degree of individualized citizens. According to previous studies carried out by the Chinese Academy of Social Sciences (Ma Chunhua et al. 2013;

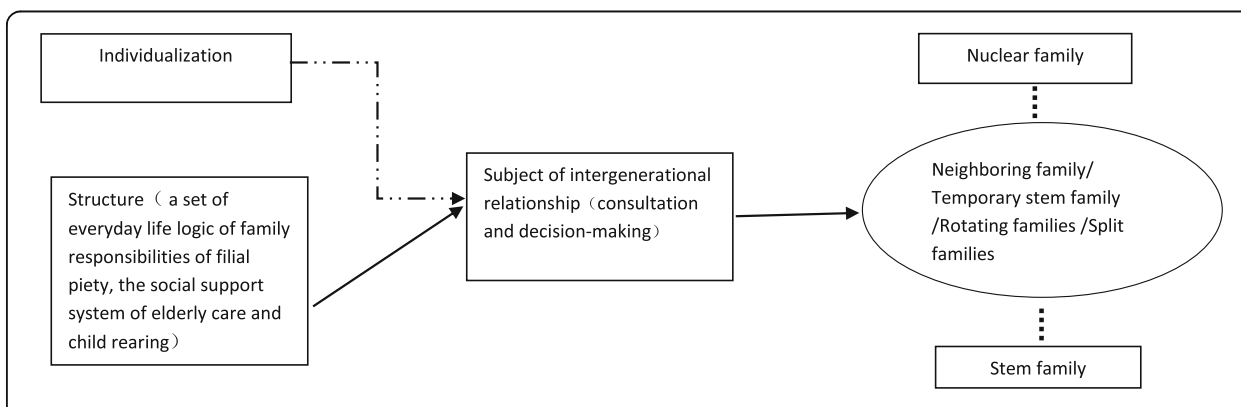

Fig. 1 The logic behind the change of family intergenerational relationship during the transition period 
Tang and Chen 2012; Ma et al. 2013), however, Guangzhou is also a very traditional city with relatively intact family or clan system, thus fully reflecting the complexity and diversity of the intergenerational relations. In July 2012, the author conducted 31 in-depth interviews in Guangzhou and visited various places in the local residential environment to widely observe, listen, and chat with Guangzhou citizens in order to understand the main variables that affect the intergenerational family relations. Second, the author did some in-depth investigation in her community and did some surveys with childhood friends, classmates, and friends. Besides, as a parent of a preschool child, the author has been able to get in touch with many families in communities of Beijing to observe the intergenerational relationship between adult children and their parents in modern families.

Robert Yin pointed out in his "sequential interview" that, unlike with sampling logic, case logic occurs one by one and step by step to accurately understand the issues that we need to study. The result of the previous case will help to bring out the questions we need to ask in the next case. The goal of a case study is "saturation," that is to have a comprehensive understanding of a problem (Small 2009). This paper follows this logic of study, and based on problem and theory, it continuously expands and analyzes cases, and mutually observes the cases from macro and micro viewpoints (Lu and Li 2007).

In order to grasp the unity and dynamic of the obtained interview data and to show the data's diversity and richness, this paper uses the combined methods of category analysis and scenario analysis.

\section{Evolutionary intergenerational family relations in different intergenerational living arrangements}

The nominal "living together"

The common perspective and method used in the study of intergenerational living arrangements is to classify the families according to the types of nuclear family and stem family, and whether two generations live together or do not live together, and measure the changes of their respective proportions. This research perspective and method is presumed by stable intergenerational members of the family and intergenerational relations; however, the temporality of members and the flexibility of intergenerational interaction are neglected.

Usually, the "living together" or "stem family" in demographic surveys takes, as the basic unit, people who live together commonly referred to as "household." Thus, these surveys may overlook the length of time and the members who live together. The author found that unlike the relatively solid traditional forms of intergenerational relations, the two intergenerational living patterns of living or not living together are becoming divided into more types, with intergenerational living arrangements are becoming more diverse and flexible, and thus the content of the intergenerational relationship may differ for the same nominal "living together" or "not living together" categories.

\section{Traditional stem family and temporary stem family}

Ms. A's husband is the only child in his family; his parents are divorced when he was very young; his mother brought him up and was not remarried. Ms. A's husband works in Shanghai as a marketing professional; he is busy with work all the time and spends 
little spare time at home. When Ms. A became pregnant, her mother-in-law move to the house in order to look after the family, since then the mother-in-law embarked on the road to "live out her life in retirement." Ms. A's mother-in-law told the author that she had no intention of leaving this family, since she is divorced and has only one child, and according to tradition, sooner or later she will live with her son's family. Normally, the young couple is kept busy by their business, and the mother-in-law stays at home to take care of their child and the housework.

Case B has the same external form of two generations living together as is the true in case A, but for the case B family, the son and daughter-in-law temporarily moved to live with an elderly parent in order to look after the individual who had an unexpected stroke in May 2011. Although they thought of hiring a nanny, the expense was a bit difficult to afford, and a desirable nanny is also hard to find. The daughter-in-law told the author that now that the elderly parent is in a functional recovery period, the young couple plans to return to their own home once the elderly family member has fully recovered, and she hope that day comes sooner rather than later.

As seen from the external form, cases A and B are both cases of families choosing to live together, namely, the stem family according to the population census. But after a careful analysis, it is found that there are big differences in these two types of intergenerational living arrangements. First of all, from a time perspective, case B is temporary situation, with families living together for a short or indeterminate time, while case A is relatively stable and lasting situation. In case $\mathrm{B}$, the younger generation plans to return to their "little own home" after the elderly individual's recovery. The time of living together in case A is relatively long, because the mother-in-law and daughter-in-law subjectively acquiesced to this traditional living pattern. Secondly, as seen from the reason for living together, case B is more in order to take care of the disabled elderly family member, reflecting immediate intergenerational support and the functional nature of living together.

Once this functional requirement does not exist, such as once the elderly individual has recovered or no longer needs care, the two generations will live apart. Although case A inevitably has functional characteristics due to mutual help occurring based on long-term living together, its nature is more based on the reciprocal culture of filial piety. In case A, the daughter-in-law told the author that she had envisioned the situation before marriage, since her mother-in-law has only one son, and unlike for other families with multiple children, sooner or later she knew she would have to adapt to this situation. Thirdly, the power structure of the two intergenerational living arrangements also differs. In case A, usually there is only one center of power. The parent's generation continues to lose power to the younger generation, especially the daughter-in-laws. The younger generation holds the decision-making power for the family; meanwhile, they have discourse power (that is, they have the final say) for their small family, and the older generation is mainly responsible for the family's logistics and their own daily lives. In case B, since they are only living together temporarily due to the illness and the need to care for the elderly, etc., the power of the family is often scattered and the absolute center of power is difficult to discern; neither of the two generations is strongly identified with this particular intergenerational structure. Instead, this family form is more determined by the family life cycle and particular family events. 


\section{Rotating families and split families}

The traditional stem family and the temporary stem family can be distinguished by the different forms of intergenerational relationships in a common residence according to the amount of time of living together. Rotating families and split families describe the diversity of living together from the different constituents of the intergenerational relationships.

The author encountered several cases that are similar to the pattern of intergenerational relations of case $\mathrm{C}$ in Beijing residential communities. Since the young generation of the family, especially those living in major metropolitan areas, such as Beijing, works from dawn to night, their child is cared for by the child's grandparents since the child was born. But unlike traditional practice, the child is looked after by grandparents on both sides in turn; generally rotating care once every 6 months. The intergenerational pattern of case $\mathrm{C}$ has been practiced for a decade, and if one side of the grandparents is unable to meet the rotation schedule, they will immediately negotiate to find a temporary solution. For example, one time, the maternal grandparents needed to look after another grandchild in the USA for a year, the family reached an agreement that the paternal grandparents will fill in for the vacant 6 months. In the following year, the grandparents from the mother's side conscientiously made up for the 6 months they were absent by taking care for the child for a consecutive 12 months.

Case $\mathrm{D}$, as case $\mathrm{C}$, is regarded as a co-residence pattern by two generations in the census. But unlike case $\mathrm{C}$, in case $\mathrm{D}$, the elderly grandparents do not live together with the younger generation, but live separatively with different children. The elderly grandparents in case D came to Guangzhou in the 1990s after their daughter-in-law became pregnant. Both of their children worked in Guangzhou. In order to make their children comfortable while working, one grandparent lives in the son's house while the other lives in the daughter's house; each helps one of their children with child rearing and housework, and the grandparents only see each other at the weekends. It happened that their son and daughter had a boy and a girl, respectively, so the two grandparents divided their work according to each grandchild's gender.

Seen from the external forms, the intergenerational living arrangements of "rotating" and "split" are both cases of two generations living together, so they are likely to be defined as a stem family and categorized as living together. But analyzing the composition of the intergenerational relationship in the two intergenerational living arrangements, we can find that these intergenerational living arrangements has changed greatly from the traditional form of having two or three generations living together, as was true under the traditional patrilineal Chinese system. Specifically, in the traditional stem family, parents and adult sons usually live together. In cases C and D, however, the constitution of intergenerational relations no longer follows the traditional patrilineal rules, and changes frequently. For example, in case $\mathrm{C}$, the grandparents from both sides of the family can come and live together; as for when or how to live together, this is a negotiation with the two or three sides discussing the issue and eventually forming a relatively stable pattern. In case D, unlike the family of "living together in turns," a pattern inherited from the traditional family separation system, grandparents are separated as a result of child rearing, so the grandparents' living arrangements become flexible-grandparents can determine how to divide the work and whom to live with according to grandchildren's gender, etc. In order to differentiate from the case of families "living 
together in turns," which is based on providing for the aged, the author will hereby name this kind of family "rotating family." This type mainly occurs to support child rearing.

Different intergenerational spatial distances in families that are "not living together" Spatial distance is a more detailed indicator reflecting the external manifestations of intergenerational family relationships. Most of the previous studies only divided the spatial distance between parents and adult children into two types of living together or not living together. In fact, intergenerational spatial distance has a significant influence on intergenerational family relations when two generations do not live together. The intergenerational relationship between an adult child who lives close to elderly parents and an adult child who resides in another city will be very different. If we ignore the different spatial distances under subtypes of not living together, we cannot have an in-depth understanding of the intergenerational family relationship.

Neighboring family is a typical pattern of intergenerational living arrangement in which elderly parents and their children do not live together and illustrates a unique intergenerational relationship.

\section{Neighboring family}

Case E is one example of the "neighboring family" the author had encountered. The man of family $\mathrm{E}$ told the author that at first he also respected the traditional patterns of intergenerational relations and thought that as an adult child he should let his parents enjoy the happiness of a family reunion, and therefore he bought a big house so that both families could live together. However, after living together, they found that the contradictions of two generations were everywhere, and both generations lived under a heavy psychological burden; finally, they had to sell the big house and bought two small houses in the same living community, so that the two generations could live separately but look after each other when needed.

Some similarities exist between the intergenerational relationship in this living arrangement and that of the stem family. That is, the functional support between generations is not weakened by spatial distance. In case E, whether the parents or adult children are facing difficulties, such as taking children to and from school or the elderly require care because of illness, the other party will come to help without any hesitation. The two families often visit each other, and if the children are too busy to cook, the parents will help with cooking or invite the children to dine together at the parents' house. Some scholars have called this type of intergenerational living arrangement "temporary stem family" (Yao 2012). It has a similar function to a stem family, but is not exactly the same. Each of the generation has a certain amount of independence. Besides mutual help, each generation has their own independent freedom and space. The elderly in case E told the author that "life became freer after living separately. I can now invite some old friends to our house, which was unthinkable in the past." It is probably because of such independence, the spatial separation did not create a rift in intergenerational relations; to the contrary, the connection of function and emotion increased. Compared with the intergenerational relationship between two nuclear families that live far apart, the intergenerational relationship between the neighboring families is very different. 


\section{Skipped generation family and nuclear family}

The author also found in the survey that in the city, there exists another type of intergenerational relationship observed in two different family forms. With the implementation of two-child policy, this pattern of intergenerational relationship is becoming a family strategy.

When the granddaughter in case F was born in 2009, her parents were busy with work. The paternal grandparents came to the house right after the child was born and helped in taking care of the child and housework. The child's father is the only child of the elderly couple, and he is qualified to have a second child. Once the selective twochild policy was implemented, his mother wanted to have another grandchild right away, and his wife also thought that the child would feel lonely without a brother or sister. After the grandson was born in 2014, the whole big family started to live together. The grandfather was responsible for picking up the granddaughter from kindergarten and preparing meals, while the grandmother was in charge of looking after the newborn grandson. In 2015 when the granddaughter needed to go to primary school, the couple found a good school in the downtown area; however, they could not afford the cost of renting a big house, so finally they had to rent a small one. The narrow space could not accommodate six people from the three generations, and after discussion, they decided that the two elderly and the grandson would live in the original house, which is slightly remote, and the younger generation and their daughter would live together in the new rental house.

Due to the children's schooling and expensive house prices/rent, the original big family with three generations living together split into two families. Seen from the external form, one is a skipped generation family of grandchild and grandparents, the other is a stem family with spouses and minor child living together. Although the adult children do not live with their parents, the intergenerational relationship between the two families is very different from the intergenerational relationship of the traditional empty-nest family and the nuclear family. Because they are raising the children, the two families have very frequent and close interactions. In case F, six people of the family gathered together on weekends to form a weekend stem family with three generations, while on weekdays they split into two forms of skipped generation family and nuclear family. And even if they are normally split into two families at a certain spatial distance, the intergenerational link is still very close. As long as there is time and opportunity, the adult children will go to visit their parents and son, bring some daily necessities, or communicate via phone and video. The author has met several cases similar to case F when conducting field research and found that they chose to be a skipped generation family and nuclear family only as a last resort, usually because of children's education or due to parents' busy work; other reasons include housing issues or problems with an intergenerational relationship.

Some families only have one child, and then they formed a skipped generation family and a conjugal family.

\section{Evolutionary intergenerational family relations}

From the above analysis, we can see that intergenerational living arrangements in modern cities have become more and more diversified. In addition, the conversion between the various forms of intergenerational living arrangements is quite common and frequent. As 
in case B, before the elderly family member's illness, they were two nuclear families; later, they formed a "temporary stem family" due to taking care of the elderly. The wife in case B told the author that they will return to their own small family when the elderly has recovered, and thus the temporary stem family will be restored to the original two nuclear families. But she also told the author that if the elderly need a longer time to recover, she might have to stay and to look after the elderly, and let her husband return to their own family first, since it is closer to his working place and he can also help their son's family which lives nearby. When other brothers and sisters visit the elderly parents at the weekend, she would go back to her own family for a while. Thus it can be seen, just in a few months, the daughter-in-law in case B may experience living in forms of nuclear, temporary stem and weekend conjugal families. The daughter-in-law in case B feels uncertain about the future, saying, "So far we can still handle it, and parents do not want a stranger to come to the house... what matter is what comes after... I will think about it later when it's approaching." That means the intergenerational living arrangements in case B may still take various forms. This makes the intergenerational family relationship more diversified and transitory.

Some scholars have advocated combining family structure and family life cycle to analyze intergenerational living arrangements (Yang and Shen 2000). This author believes that family life cycle will inevitably affect intergenerational living arrangements in modern cities, but that alone is not sufficient to explain such frequent and flexible intergenerational flow. Intergenerational living arrangements are affected by family life cycle that are usually irreversible and immutable, while the changes to intergenerational living arrangements discussed herein are usually flexible and short-lived, and families may revert to the original form of the family's life cycle after a certain time. In other words, this change of intergenerational form is more like a redifferentiation of the intergenerational form within a family life cycle. This evolution in the intergenerational relationship is relatively similar to the "latent kin matrix" proposed by some scholars-a network that is shifting, but can inspire and deepen the close kinship at any time (Riley 1983). One of the significant characters of this network is that it is very responsive to the needs of its members.

So what factors led to the evolution of intergenerational relations during China's transition period? What is the logic behind it? We will analyze these questions in the next section.

\section{Logic behind the evolvement}

\section{The individualistic trap of three generations living under one roof}

In Chinese society, when three generations live under one roof, it was seen as an ideal state of intergenerational relations since it reflects both "providing for the elderly" and "filial piety." The significance of this intergenerational living pattern is mainly reflected in two aspects: first, the elderly can be taken care of and enjoy the happiness of a family union, and simultaneously spiritual emptiness and loneliness can be reduced; second, children can receive real affection and care, while adult children can inherit their predecessors' life wisdom and concentrate on work with no worries (Hu 2004). This intergenerational living pattern is not only in line with the filial piety requirements of Chinese culture, but also consistent with economic rationality. But how can three generations live together under one roof when they have different backgrounds, lifestyles, 
and personality characteristics, and how can they create a harmonious picture? This has always not been an easy task. Especially under the influence of the second modernity, the individualistic value has been strengthened. Although the issues of "degree of Chinese individualization," "the cause and effects of individualization in the rise of China," and "differences between Chinese and Western individualization," etc. are still controversial in academic circles, but there is no denying that today's Chinese society is experiencing a transformation in individualization. Yan Yunxiang has pointed out that individuals in the Chinese society who break away from family or family organization due to the influence of the national political movement in the collectivization era, are further discovering the self and reshaping the self under the dual effects of the market economy and changing national policies. Although China's individualization does not emerge from the premise of individualization seen in Western European countries such as welfare state, cultural democracy, political liberalism, and classical individualism, globalization of the market economy and consumer ideology have provided flexible choices in occupation and labor market with a high degree of mobility, combined with interventions from state power, resulting in the traditional family system weakening or being impacted, to form a culture that emphasizes risks, intimacy, self-expression, and self-dependency (Yan 2012). The individualization trend is bound to bring new influences on family intergenerational relationships, and the author can certainly confirm this trend in in the research.

Parents and adult children live together under one roof, with humility and filial piety on the part of adult children and grandchildren, and this has been the traditional form of intergenerational relations in China. However, the author encountered several different cases, finding that this form of intergenerational family relationship is becoming increasingly fragile and changeable as parents and their offspring are becoming increasingly independent and parents have declining authority. Yan (2012) believes that when wealth is no longer continuously handed from the previous generation to the next, the younger generation may accumulate more wealth than the last generation through their efforts or opportunities at a relatively young age. The younger generation thus begins to demand social status corresponding to their economic status. With increasing economic capability and consumer desire, the younger generation has a strong motivation to live separately from their parents. From this survey, the author found the older generation, who have lived under the reform and opening up policies, accumulated significant disposable wealth at a young age. They are also influenced by the culture of intimacy, self-expression, and self-reliance, therefore prefer to live separately from the younger generation.

For example, in case E, three generations used to live together, but conflicts often occurred. According to the mother-in-law, conflicts mainly concentrated on two aspects: living habits and children's education. She was a frugal person and not happy with her daughter-in-law's extravagance and waste, but the daughter-in-law thought of herself as financially independent and believed there was nothing wrong with spending her own money. Especially regarding the children's education, the mother-in-law, a retired teacher, had her own beliefs about the value of educating children in the traditional style, and this differed from the modern educational theory advocated by young generation. The mother-in-law felt lost because her education theory was not recognized, while the daughter-in-law felt both her education theory and life were being interfered 
with by the mother-in-law. The parents-in-law happened to be retired cadres and therefore they had some savings that could make up for the price difference in moving to a new house. After consultation, the original stem family split into two nuclear families.

It can be seen that the individualistic ideas and values of the child and parent generation are constantly enhanced with improvements to education and economic conditions brought about by China's reform and development, so both younger and older generations are hoping to achieve individual freedom and live in the life trajectory that they determine for themselves, and family life has become an opportunity to express self-identity and self-invention (Cherlin 2008). The elderly in case E told the author that, "separate living is good for both two generations. We have our own space and freedom, and more contacts with old friends." Thus it can be seen, the elderly of three different generations not living under one roof can contribute to their social communication, talking, leisure, and sports health; meanwhile, they can obtain self-esteem by independent self-living and obtain emotional support from family and friends. The modern elderly seek exactly this: expression of their individualization. The younger generation is also free from conflicts between daughters-in-law and other people in the family, and arrange their life according to their own ideas to meet the needs of self-identity and self-creation. Thus, the individual needs of different generations are satisfied by not living together.

\section{The plight of the nuclear family}

Although the individual needs of the main body of intergenerational relations are satisfied by not living together, the author found in the survey that forming this intergenerational living arrangement is possible because the elderly are still "young" and there is no need to care for children's upbringing or to provide care for the aged in the family; or, as in case E, the two generations have enough economic strength to afford two houses in one or adjacent residences in the same community. This living pattern does exist, but in Chinese cities in the transition period, it is more common to choose other types of temporary or flexible intergenerational living arrangements given the structural constraints; these include temporary stem family, rotating family, split families, skipped generation families and so forth. Especially, the extension of average life expectancy and the implementing of the two-child policy have extended the entire family's time for providing care for the aged and children, and family becomes the only choice when people face such social risks.

Structural constraints mainly come from the set of life logic regarding family responsibilities in a culture of filial piety, as well as the absence of any related social system for pension or childcare.

First, consider the imperfections of the related social systems of pension and childcare. The intergenerational family relationship is a bidirectional relationship between different intergenerational family members, with upward care primarily related to providing care for the aged, and downwards to childcare. Unlike the "emotion-oriented" intergenerational relationships found in Western countries, urban Chinese adult children and parents still have a strong functional interdependence; in some areas, it even has been strengthened. This is closely related to the imperfect social system, which provides little support for elderly care or childcare in the transition period. In China, the 
impact of state strength on the construction of intergenerational family relations is immeasurable. Western countries, such as northern European countries, emphasize the importance of national welfare, or reducing the family burden through socializing family functions. While China has been committed to changing family into a "social unit," a unit suitable for industrialization and marketization, at the same time, the state lacks the necessary social welfare system to support the family in "modernization" (Yao 2012); especially since the 1990s, the state has constantly withdrawn from people's private lives, so people have to face the reality that the state has withdrawn from the private sector and left the various relevant functions to family again (Liu 2016). The intergenerational family relationship thus has become the last bastion for the individual to resist risks.

For instance, in case B, although a comprehensive old-age insurance system and a health care system have been established in cities, the functioning of these systems still remains limited. In China, long-term care for the elderly is still primarily provided by the spouse or adult child. The daughter-in-law in case B told the author that "when the elderly family member is sick, the younger generation is certainly involved in any case; you have to keep watching in the hospital all the time, even if you've hired a nurse attendant you have to keep one eye on things from time to time since nowadays the quality of the nurse attendant is not that good. When the elderly family member is convalescing at home, we children have to be more worried about their care." "A housekeeper? As a salaried person we really can't afford it; besides, it's very difficult to find a reliable housekeeper. Even though we hired one, we adult children have to worry about the care and often come over to take a look."

This shows that China's current old-age insurance system (including pension and health care) can only ensure the basic survival needs of the elderly; family still play a major role when the elderly need to be tended to because of illness, especially when need long-term care. As with the daughter-in-law in case B, although she felt discomfort and was frustrated, she still had to choose the intergenerational living form of temporarily stem family. From her helpless expression, we can also observe that this form of intergenerational living is flexible and variable, and it may change according to the situation of children and the elderly at any time.

Such basic care is also true of childcare. In traditional societies, the family is the main provider of family welfare; childcare is the family's main function and responsibility. In the planned economy era, in order to encourage women to enter the labor market, the government set up a public childrearing system in danwei for their staff and workers to access free or low-cost child care and education services. After the reform and opening up, the government began to tilt all resources towards economic development. In addition to implementing a family planning policy system that controls births in a family, the state gradually withdrew from the family welfare system. Whether in urban or rural areas, childcare responsibilities have returned to the family, and childcare services have become market-oriented. Although in recent years the government has planned relevant policies to accommodate the serious mismatch between supply and demand of childcare services, the childcare policy is aimed primarily at only children and children facing difficulties; in addition, the strength of support is very limited. For most children, the government mainly provides compulsory education and health care. Caring for children, especially preschoolers, has become a heavy burden on the family (Ma 2014). 
In addition, the development of the market economy brought about an open society, and the work stress of the younger generation is growing due to increasing pressure from employment and competition, so the heavy responsibility for caring for the family can only be passed on to the elderly.

For example, with the case $\mathrm{C}$ family, the younger generation are all working in companies with fierce competition, and as described by the daughter-in-law, "you will be replaced immediately if you slack off even a little." "I also think it's better to bring up the child by myself. First of all, it benefits the child; secondly, it can reduce conflicts between the two generations, but there is really no choice. Expensive house prices and high cost of living and education expenses made it impossibly for me to return to home as a housewife." The young urban generation work from dawn to dusk every day, and sometimes have to work overtime, so they have to ask the elderly help to take care of children and housework. At first, in case C, only the parents from the father's side came to help and they intended to stay only until the grandson went to kindergarten. But due to lack of formal childcare services in the city, the grandchild can only be taken care of by family members when he is not in school, since the adult children have to go to work early and come back late every day. Thus, this family responsibility inevitably passed on to the elderly. Fortunately, the adult children can understand the discomfort and difficulty of the elderly, so they found a compromise approach of turntaking by the elderly from both sides (sometimes this is also a family strategy of resolving conflicts). The daughter-in-law in case $\mathrm{C}$ thought the biggest advantage of doing this was to reduce the burden and complaints from the elderly grandparents, and further, to avoid some family conflicts. "The elderly can be bored easily when staying here for a long time without their own space; in such a situation, conflicts can easily arise." "Living in this way can let the elderly have a chance to take a break. It's good for both generations." In an interview with the elderly, the elderly also feel that this pattern is better than long duration living together, "This is the only solution, we certainly want to have our own freedom and living space, but working and taking care of kids at the same time is also hard for our children, so we had to help." "This is still better than those families who have to live together for a long time. At least, we have the chance to take a break. We can go back to our home or go travel sometimes." "When staying in here we know that we'll leave soon anyway, we can't manage everything. So we just leave it for our children, and thus having less conflicts."

These are the institutional reasons, which reflect the great power of the state in shaping intergenerational family relations. However, institutional factors are not enough to explain why elderly parents still work hard to help adult children with housework and childcare in some cities where rich pension resources exist. Why does a trend similar to the Japanese housewife, someone who eases pressure on family upbringing, not appear in China? Why do responsibilities for childcare for the family still mainly fall on the elderly, and providing for the aged, especially for care for the elderly unwell, still remain the responsibility of adult children? Although functionality is the major purpose of intergenerational family relations in the transition period, not all the intergenerational relations of intimate and mutual family benefits are derived from practical considerations. In fact, the author found that intimate contact and mutual assistance between generations also originate in the Chinese culture of filial piety, which outlines family responsibilities and obligations. 
For example, the daughter-in-law in case A invited the mother-in-law to live together with her family. In addition to considering the pressure from work and childcare, the daughter-in-law also took into account the duty of filial piety. The daughter-in-law of family A told the author that when she decided to marry her husband, she had already taken into account this point, since her husband is the only son of the family. According to the traditional Chinese custom, it is clear that the couple will live with the mother-in-law and look after her in her old age; at the same time, the mother-in-law also realizes the continuing efforts from adult children will securely provide for her old age, and her hard work in helping the family will benefit family achievements and continuity. As the mother-in-law said, "We become old and useless, so the greatest desire of the older generation is to do our best to help children focus on work for achievements, and to help grandchildren growing up healthily and safely." The profound impact of the reciprocal filial piety culture can be found in both case $\mathrm{C}$ of the rotating family and case B of the temporary stem family.

The author found that, although times change, the culture of reciprocal filial piety is still deeply rooted in the individual's subjective consciousness, providing a cognitive sense of security, belonging and purpose of existence for individuals facing social risks (Zhao and Kang 2010). Data from the China's Five-city Family Structure and Relationship Survey on Guangzhou also verified this point. It shows that $99.8 \%$ of people choose in favor of "children should support their parents"; meanwhile, there was no significant difference in sense of filial piety for different age groups. Particularly noteworthy is that $74.47 \%$ of respondents born after the 1980 s choose "strongly agree" with "children should support their parents," accounting for the largest proportion in all age groups. The survey shows that filial responsibility has not significantly weakened over time, at least at the cognitive or emotional levels. On the pension issue, whether it is for the individual's own pension or their parents', more than half of respondents think children should bear the responsibility of supporting the elderly; only $3.4 \%$ choose "parents should spend their own money to hire someone to take care themselves." This shows that "raising children to provide against old age" and expecting children's filial piety are still common social psychology. Adult children not only believe that they have their own duty to fulfill the filial responsibility to their parents, but also expect their children to do the same, and thus filial responsibility still occupies an important position in people's intergenerational norms (Shi 2013).

\section{The logic behind the evolution}

From the above analysis, we can see that factors at the individual and structural levels work together to constrain changes in China's intergenerational relations. However, unlike in developed Western countries, the changes in intergenerational family relationships in China are more affected by the special social transition and cultural traditions; the forces at the structural level are greater than those at the individual level. The diversified intergenerational living arrangements that exist in Chinese cities at this stage provide strong evidence.

Having three generations live under one roof has brought troubles to individuals' freedom and autonomy, and thus is no longer the ideal or the reality in the era of individualization. Whether older or younger, each generation hopes to arrange their 
lives in accordance with their own ideas and to have their own independent spaces and freedom. But in many cases, forming a nuclear family is still difficult in reality. Reasons include the elderly have to help raise grandchildren due to a highly competitive labor market and imperfect childcare policy; the family cannot afford expensive housing to live separately; the elderly may be sick or in need of care so that most adult female children cannot "return to their stem family"; as well as the expectation of the "return" of reciprocity through filial piety. According to the analysis of some scholars, this return is a tool used by individuals to seek self-development in the era of individualization (Shen 2013), while some scholars believe that it still contains a factor of pure affection (Liu 2016). In short, the nuclear family will face various dilemmas due to social risks, and these risks can emerge at any time. Although individuals may be unwilling to live together, the two sides of the intergenerational relations will determine the meanings of the individual and the relationship within their relations. For example, parents will consider the needs of kinship, pension, or family continuation; adult children will consider the responsibility of filial piety and self-development pressures, and then, they find a variety of strategies to deal with relationships to ensure their needs are satisfied. Both sides of the intergenerational relationship are trying to find a balance between the structure and the individual, but are influenced by the system and a set of logic on the family responsibilities within the filial piety culture. The actual outcome is often that consideration of structural aspects will override individual pursuits, thus making people only choose some special intergenerational living arrangements that can be described as existing between the forms of stem and nuclear, such as the temporary stem family, skip-generation raising family, neighboring family, rotating family, and split family, thus forming an ever-evolving intergenerational relationship during the transition period.

Yan (2012) affirmed the process of individual socialization in China and points out that it is different from the Western model. China's party-state management, with its lack of welfare state institutions and classic individualistic culture, made China's individual transformation bear the features of pre-modern, modern, and post-modern simultaneously, and Chinese individuals must deal with all of these conditions. In addition, the tradition of long-term support of Chinese family members, and the key role of social relations in shaping and re-shaping personality, have made the individualized process take a different path towards development (Yan 2012). This assertion is confirmed by the author's research. Most parents and their adult children prefer privacy, freedom, and independence as the best choices. This is especially true for the older generation, who experienced reform and opening up. Compared to support for finances and life care, they have greater expectations of free space, harmonious relations, and emotional comfort. But, most of them eventually are restricted by the social structure and have to push individual pursuits to second place.

By using the above chart, the author tried to outline the logic behind the change of intergenerational family relationships in the transition period. It can be seen from the chart that the forces from both the structure and the individual together constrain the intergenerational family relationships in the transition period, which is consistent with the description of the Luscher theoretical school. But in China, during the transition period, the strength from structure is stronger than the strength from the individual (dotted arrows in the chart indicate the impact of individual on intergenerational 
relationships, showing the strength of individual is weaker than the strength of structure). Influenced by a set describing the everyday life logic of family responsibilities for filial piety, as well as the social support system of elderly care and childcare, while still not willing to give up the pursuit of individual goals, most subjects of urban intergenerational relations are forced to choose a certain form of intergenerational relationship that exists between nuclear family and stem family at different times and spaces, thereby forming flexible intergenerational relationships. Thus, to accurately grasp and understand the characteristics of intergenerational relationships during the transition period of China, we need to place it in the context of China's special individual and structural choices.

\section{Conclusion and discussion}

The intergenerational family relationship is the longitudinal presentation of family relationships. Since the 1980s and the 1990s, economic and social transformations have brought great changes to Chinese society. Intergenerational relation, as one of the two basic axes of family relations, has also been profoundly changed.

As the external manifestation of intergenerational family relationships, the pattern of intergenerational relationships, namely, intergenerational living arrangements, is one of the most direct and most objective dimensions, from the perspective of external observation, of the intergenerational family relationship. Beyond the classic "extended-stemunclear" morphological classification framework of intergenerational relations, this paper explored the characteristics of China's intergenerational family relations during the transition period and its formation mechanism based on the practice of the family's everyday life.

This study found that diversified intergenerational living arrangements have become a dominant feature of intergenerational family relations in urban China, and this reflects the evolution of intergenerational family relations. Seen from the length of living together and the composition of intergenerational relationship, under the same nominal "living together," there are actually different patterns of intergenerational living arrangements. It is the same situation with "not living together". Different intergenerational spatial distances present various contents of intergenerational relationship. Under the dual impact of structure and the individual, and according to different times and spaces, the main subjects of intergenerational relations will form different types of intergenerational relationships. The main subject will first search for the meaning of the individual within the relationship, and then look at various social situations and conditions which can cause individual and collective behaviors to happen; finally, they will make comparisons and choices between different forces in order to find the best strategies and solutions. People often face a series of selective conflicts and make the choice between pursuing individual independence and complying with family responsibilities. This constant comparison, with trade-off and selection, contributed to the formation of the evolving characteristics of intergenerational relationships during the transition period in China.

The great power of government in shaping this feature of intergenerational relationship is not to be ignored. Since the 1990s, as the state continues to withdraw from areas of private life, many of the state responsibilities associated with elderly care and childcare have been returned to the family. In the current situation of compressed 
modernity, individuals in the family have not been completely liberated from the bondage of the pre-modern filial tradition, and again are further embedded in intergenerational relationships under the post-modern risk pressure (Chang 2010). Families, the only place that can afford the burden of elderly care and childcare, have become the last bastion of the individual in a risk society as a site to resist risks. The older generation, who experienced reform and opening up, has achieved financial independence, but has not achieved complete independence. In China, the current oldage security system can only meet the basic survival needs of the elderly, and thus sick care for the elderly is still mainly borne by the family. Childcare is also the responsibility of the family. The market economy has pushed issues of childcare, housing, etc. into the market, and coupled with increasing employment pressure and pressure from competition, the younger generation has borne huge pressures in the workplace. As a result, the heavy task of childcare can only be passed on to the older generation.

In such circumstances, reciprocal filial piety has the solid ground of continuation. Reciprocal filial piety emphasizes the two-way relationship of "loving father, faithful son." Where does the child's deep affection for his/her parents come from? Only when parents' love is given to children as deeply as possible, can deep affection form and filial piety to parents will arise voluntarily from children. Parents should use their love to gain filial piety, while children should support or pay back their parents' love with filial piety (Yeh and Yang 2009). This generational concept based on the principle of fairness happens to fill the gap between intergenerational family needs, specifically in terms of elderly care and childcare, and has become the internal driver of intergenerational mutual aid. These two great forces from the Chinese system and culture has caused family members to only seek individual independence and space in the cracks in the existing structure and to choose flexible intergenerational living arrangements, thus establishing unique and evolving family intergenerational family relations during the transition period in China. Due to the limited scope of this case study, the author cannot analyze all types of intergenerational residential arrangements during China's transition period. However, the diverse intergenerational living arrangements that the author has illustrated prove that the change in Chinese intergenerational family relationships is not as predicted by classical family modernization theory, which has previously described an unidirectional propulsion from traditional extended family to nuclear family/conjugal family, families that maximize equality and individualism (Ma Chunhua et al. 2013). Instead subjected to structural and individual factors, various evolutive intergenerational patterns have emerged between stem and nuclear family, such as temporary stem family, rotating family, split family, neighboring family, and skipped-generation family.

When three generations live under one roof, social problems are privatized and costs for family care are passed on to the elderly or women. This ideal model of intergenerational living is in decline in modern society as a result of growing individualization. However, not living together does not mean the collapse of intergenerational relations. Scholars should further study how to reduce the problem of elderly and child care relative to the distribution of family duty and money, as well as how to build relations between parents and children more based on spontaneous intimacy. These two issues will continue to be of importance to scholars who study intergenerational relations during China's transition period. 


\section{Competing interests}

The author declares that he/she has no competing interests.

\section{Publisher's Note}

Springer Nature remains neutral with regard to jurisdictional claims in published maps and institutional affiliations.

\section{Received: 1 November 2017 Accepted: 4 December 2017}

Published online: 20 December 2017

\section{References}

Beck, U., and E. Beck-Gernsheim. 2011. Individualization, Li Rongshan, Xuan Fan, Huiqiang Zhang (trans.). Beijing: Peking University Press.

Bengtson, V., R. Giarrusso, J.B. Mabry, and M. Silverstein. 2002. Solidarity, conflict, and ambivalence: complementary or competing perspectives on intergenerational relationships? Marriage and Family 64 (3): 568-576.

Bengtson, V.L., and R.E.L. Roberts. 1991. Intergenerational solidarity in aging families: An example of formal theory construction. Marriage and Family 53: 856-870.

Chang, Kyung-Sup. 2010. Individualization without individualism: compressed modernity and obfuscated family crisis in East Asia. Intimate and Public Spheres 3: 37-62.

Chen, Jieming, and Qi Chen. 2016. Parent-child socioeconomic statuses and co-residence: an analysis of living arrangements in China. Sociological Studies 1: 73-97.

Cherlin, A.J. 2008. Public and private families: an introduction. New York: McGraw-Hill Humanities.

Connidis, I.A., and J.A. McMullin. 2002. Sociological ambivalence and family ties: a critical perspective. Marriage and Family 64: 558-567.

Goode, W.J. 1982. The family, Zhangling Wei (trans.). Beijing: Social Sciences Academic Press.

$\mathrm{Hu}$, Yow-Hwey. 2004. Three generation dwelling: the myths and pitfalls. Taipei: Jyu-Liou Publishing Co., Ltd..

Kang, Lan. 2009. The change of feedback model: study on intergenerational relationship in the urban area from the perspective of generational gap, doctoral dissertation, 1-5. Shanghai: Shanghai University.

Laslett, Peter. 1972. The household and family in past time. Cambridge: Cambridge University Press.

Liu, Baojiu. 2000. Study on the change of structure of modern Chinese urban family. Sociological Studies 6: 31-32

Liu, Wenrong. 2016. The intergenerational emotion and solidarity in transitional China: comparisons of two kinds of parent-relier families in shanghai. Sociological Studies 4: 145-168.

Lu, Huilin, and Xue Li. 2007. How can we go beyond the case? From the case study to the extended case study. Social Sciences in China 1: 118-130.

Luo, Meijun. 2004. Marriage and family economy in the end of the 19th century and contemporary rural China. In New horizons in the study of family history, ed. Guogang Zhang. Beijing: SDX Joint Publishing Company.

Lüscher, K. 2002. Intergenerational ambivalence: Further steps in theory and research. Marriage and Family 64 (3): 585-593.

Lüscher, K., and K. Pillemer. 1998. Intergenerational ambivalence: a new approach to the study of parent child relations in later life. Marriage and Family 60: 413-425.

Ma Chunhua. 2014. Reconstructing the contract between the state and young families: Collective sharing of responsibility for child rearing. Youth Studies 4: 66-67.

Ma, Chunhua, Yinhe Li, Can Tang, Zhenyu Wang, and Jinqun Shi. 2013. Family change in urban areas of China during social transformation: based on the survey in five cities. Beijing: Social Sciences Academic Press.

Parsons, Talcott. 1949. The social structure of the family. In The family: its fuction and destiny, ed. Ruth N. Anshen. New York: Harper.

Riley, M.W. 1983. The family in an aging society: a matrix of latent relationships. Marriage and Family 4: 439-454.

Shen, Yifei. 2013. The individual family iFamily: individual, family and country in the process of urban modernization in China. Shanghai: SDX Joint Publishing Company.

Shi, Jinqun. 2013. A study of the intergenerational relationship between adult children and their parents, doctoral dissertation, 2-3. Beijing: Graduate School of Chinese Academy of Social Sciences.

Shorter, Edward. 1977. The making of the modern family. New York: Basic Books, Inc..

Small, Mario L. 2009. 'How many cases do I need?' on science and the logic of case selection in field based research. Ethnography 10 (1): 5-38.

Song, Jinwen. 2001. The contemporary Japanese families theory and the elderly care. Sociological Studies 5: 95-104.

Tang, Can. 2010. A review of modernization theory and its development on family. Sociological Study 3: 152-156.

Tang, Can, and Wuqing Chen. 2012. The kinship of Chinese urban families. Jiangsu Social Sciences 2: 92-103.

Wang, Yuesheng. 2011. Contents of intergenerational relationships in family and its changes in different periods of China - an analysis based on combination of history and reality. Journal of Graduate School of Chinese Academy of Social Sciences 3: 136-142.

Xiao, Suowei. 2014. Yan mu Ci Zu: The intergenerational collaboration and conflicts in child rearing among urban families in contemporary China. Sociological Study 6: 148-171.

Yan, Yunxiang. 2012. The individualization of Chinese society, translated by Lu Yang, etc. Shanghai: Shanghai Translation Publishing House.

Yang, Shanhua, and Chonglin Shen. 2000. Family change under the background of market economy and nonagriculturalization. Hangzhou: Zhejiang People's Publishing House.

Yao, Jun. 2012. Temporary stem family': the transformation and strategy of family structure in cities-based on the case study in N City. Youth Studies 3: 85-93.

Yeh, Kuang-Hui, and Kuo-Shu Yang. 2009. Chinese filial piety: an analysis of psychology. Chongqing: Chongqing University Press.

Zhao, Zhiyu, and Yingyi Kang. 2010. Social psychology of culture. Beijing: China Renmin University Press. 